\title{
Research on the Application of Intelligent Monitoring Technology for Collection of Cultural Relics Based on the Internet of Things
}

\author{
Hanwen $\mathrm{Li}^{1, \mathrm{a}}$, Yufei Wang ${ }^{1, \mathrm{~b}}$ and Kun Zhang ${ }^{1,2, \mathrm{c}^{*}}$ \\ 1. College of Ocean Information Engineering, Hainan Tropical Ocean University, Sanya, Hainan, \\ 572022, China \\ 2. State Key Laboratory of Marine Resources Utilization in South China Sea, Hainan University, \\ Haikou, Hainan, 570228, China \\ a Ihw9954@163.com, ${ }^{\mathrm{b}}$ gwmfox@126.com, ${ }^{*} \mathrm{c}$ kunzhang@hainu.edu.cn \\ *The corresponding author
}

Keywords: Internet of things; Cultural relics monitoring; Museums; Sensors

\begin{abstract}
With the development of Internet of things' technology, all fields of social life are use related technologies of the Internet of things. Using Internet of things' technology to monitor cultural relics in museums can protect cultural relics not to be damaged. In combination with sensor, numerical control system, video monitoring system, transmit data system, accept data system, processing data system and so on in the cultural relics monitoring equipment, it can monitor and control the Cultural relics' situation effectively. Make sure they are in the right circumstances, protect precious cultural relics scientifically.
\end{abstract}

\section{Introduction}

China is a country which has thousands of years of rich history and culture. Huge amounts of precious material and cultural heritage was saved until nowadays. Because of this, people can learn and discover extensive and profound ancient culture throughout these treasures. Therefore, the main task now is to protect all kinds of cultural relics that have been found. The significance of the museum is to display the cultural relics, whose main task is to manage, protect and collect these cultural relics. In the process of protecting cultural relics, it is necessary to monitor their environment. The traditional method is to detect their environment through manual inspection, but this method requires huge workload and a large number of staff, and still cannot fully protect them. With the development of science, the society has entered the intelligent era. From the proposal of the Internet of things to its rapid development, more and more fields have applied this technology successfully. The technology of Internet of things, which realizes the information transfer between objects and objects, and people and things, can be applied to the monitoring equipment of Museum cultural relics, to manage and protect cultural relics more scientifically and effectively, and provide a more suitable environment for the preservation.

\section{Overview of Internet of things technology}

Internet of things, it is connected to the Internet with facilities and equipment such as sensor, numerical control equipment, video monitoring equipment, frequency identification equipment (RFID) and so on. Through concrete agreements, to make convey information between things or person-to-thing come true.

Internet of things, it also called "A sensor network". It was born in 1999, but after Premier Jiabao Wen put forward "Touching China" in 2009, this technique began to be focused heavily. Internet of things' structure divided into three layers. The first one is Perception layer from the bottom up.Perception layer is the core part of the technique, it can collect information because of its full and accurate perception. It be mainly applied to sensor, RFID Electronic tags, the smart card and so on. The second one is The network 
layer, it can optimize Internet of things' application features. It need big coverage mobile communication network to be the technical support. It can transmission of information based on the wireless networks, Internet and TV network and so on. The third one is the application layer. It can provide a variety of applications, make recognition and perception between things, person-to-thing, play a smart role.

At present, Internet of things technology is already is used in many areas like intelligent building, intelligent electric meters, Logistics warehousing, Street lamp management. At the same time, also include traffic, medical and another domain. Its application in the environmental monitoring and security system is also applicable to museum relics' Monitoring equipment.

\section{Application of internet of things technology in cultural relics monitoring}

Internet of things technology can apply to every aspect. from the function of application and implementation, it be mainly applied to four aspects. They are Cultural relics' temperature and humidity detection system, Cultural relic location tracking system, Heritage storage management system and Cultural relics transport management system.

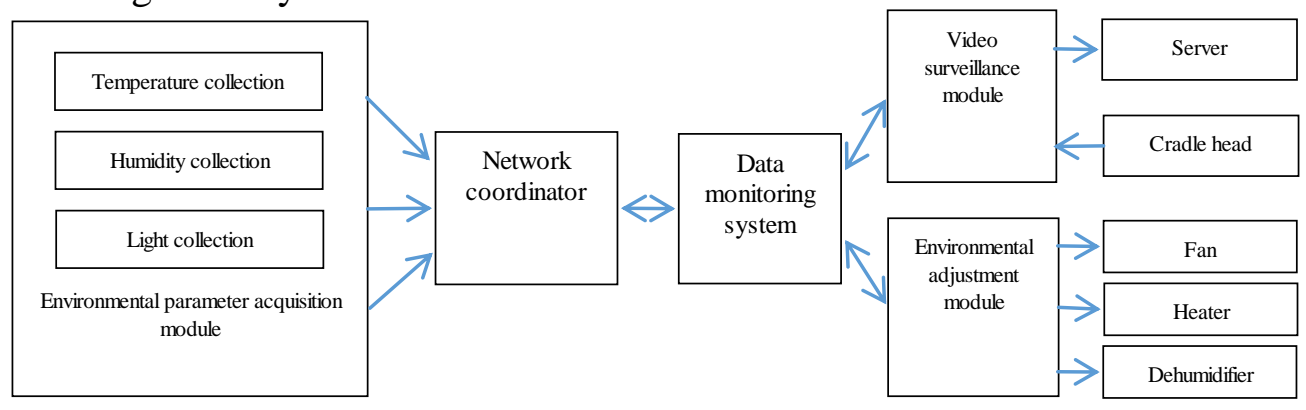

Figure 1. System overall structure diagram

Temperature and humidity Internet of things monitoring system. At the museum, there are two main aspects in monitoring of Cultural relics: Temperature and humidity. Cultural relics are different material,so the sensitivity are different.Unsuitable environment will speed up the aging speed of cultural relics, even increase the extent of damage to cultural relics. Therefore, different cultural relics' requirements and features to environment, in combination with design of sensor technology research and implement environmental monitoring equipment, control the museum's environment scientifically. For temperature and humidity monitoring, there are two things to note: The proper value of cultural relics of temperature and humidity; Control volatility of temperature and humidity in the museum. Although low temperature could reduce the rate of chemical reaction when the cultural relics be displayed or stored, it also could make some failure on Cultural relics of different materials. So, the cultural relics in the museum should be preserved at room temperature. As for the good value of humidity, too dry or wet are all bad. At the same time, different materials have different requirements for humidity. We need consider climatic characteristics of local cultural relic's storage area.

The temperature and humidity are unstable, it will damage to cultural relics. The main reason is the frequent fluctuations in temperature and humidity. It would make cultural relics shrinkage unevenness. And then make the preservation of cultural relics is difficult. Therefore, Temperature and humidity monitoring system is also need control the environment when the temperature and humidity are fluctuates continuously because of climate and other uncontrollable factors to protect cultural relics.

Temperature and humidity monitoring system connected with Internet of things technology using sensors and wireless transmission technology mainly. Real-time monitoring of the environment of the cultural relics through the sensor, and transfer the specific data of temperature and humidity to radio signal, and then to the monitoring system. The monitoring system will transfer the radio signal to the specific data, and analyze the data. The results of the analysis display on relevant staff's Mobile terminals. At last, the staff 
will dispose it. Coupling Internet of things technology to temperature and humidity monitoring system at museum reasonably, it can detect changes in temperature in time. And then measure better. In this way, the temperature and humidity of the environment in which the cultural relics are located are always appropriate. To facilitate the protection of cultural relics,reduce the output of the labor force and efficient and comprehensive protection of cultural relics.

Cultural relic positioning system. The cultural relics positioning system is mainly applied to ensure the security of cultural relics. The positioning system combines with the Internet of Things technology and confirms the position of cultural relics in real time. The possible warehouse-in and out of cultural relics make it more difficult to manage cultural relics and ensure their security. Introducing the Internet of Things cultural relic positioning system to position relics to avoid at all possible their losses or someone privately bringing them out of museums. Meanwhile, the positional system of cultural relics can also be applied to the exhibition of cultural relics. Using the positioning system to precisely locate cultural relics and show the positional information of relevant relics to provide accurate positions to tourists coming to visit relics exhibited by museums. Tourists can visit and research cultural relics they are interested in according to positional information suggested. The cultural relics positioning system combined with the Internet of Things technology works mainly through making passive RFID into self-adhesive paper labels and pasting them on cultural relics in museums. RFID can position and identify cultural relics in real time and then using the RFID reader to recognize information of tags based on the position by ZigBee Internet technology. Using ZigBee Internet technology to wirelessly transmit tag information collected by the reader to the position management system to identify the position of cultural relics in museums. The position management system can collect and aggregate received information of position and display them with the visual interface in mobile terminals of relevant staff so that they can identify and supervise the position of relevant cultural relics in real time.

By designing and implementing the Internet of things location system, the staff of the museum could check and confirm the location information of relevant cultural relics at anytime and anywhere. And monitor it. When there are some emergency happened, like cultural relics are brought out of the museum by unauthorized persons, the cultural relic positioning system can send out the alarm information in time. And then the staff can make relevant protective measures to the cultural relics in time.Stop the theft of precious cultural relics. Moreover, the cultural relic positioning system can greatly reduce labor resources. There is no need for a large number of staff identifies the location of each cultural relic frequently. During the cultural relic's exhibition, staff members are no longer required to introduce and guide tourists to the location of relevant cultural relics. This also reduces the daily workload for most staff.

Cultural relic storage management system. In the relevant work of the museum, the management of the cultural relics and the work of the storage of cultural relics are also very important. With the development of science and technology, RFID technology has also been widely applied to the museum's inventory management system and the management system of relevant staff's management system of the warehousing. However, the management of precise storage location of cultural relics still requires a large number of staff to collect them manually. This will result in a waste of human resources, which will also increase the workload of staff and spend a lot of time collecting statistics. Going through the way of artificial statistics this exposition hall of cultural relics, the concrete which is stored in the warehouse, hundreds of thousands of cultural relics in which collections on the shelf in the warehouse, and there is no doubt that this is a very difficult and demanding task.

Therefore, it is necessary to improve the existing equipment that the storage and management system which used the Internet of things technology on the basis of inheriting RFID technology. Cultural relics storage management system's other functionality that needs to be implemented are: Accurately locate the storehouse number and collection rack number of the cultural relics in order to find and manage; Update the information of the inventory of the cultural relics in real time; Manage the staff in the warehouse. 
Make cultural relics storage management system come true is similar as Cultural relic positioning system. It also uses the passive RFID electronic tag to identify and locate the location of cultural relics, and identify the tag information by using the electronic tag reader. The location information collected by ZigBee network is transmitted in the wireless to the positioning management system. The information is summarized by the positioning management system and displayed on the manager's mobile terminal. However, unlike the cultural relic positioning system, the reader needs to select a fixed electronic tag reader and install it in the doorway of various warehouses to locate the relics accurately. For the management of the staff's warehousing, the staff should be equipped with a work card with a passive RFID electronic tag. When a staff member passes through the electronic tag reader at the gate of the warehouse, the information can be identified. Thus, the staff's warehousing is managed.

Cultural relics transport management system. The museum, with its high exhibition usage or few visitors, needs to transport the cultural relics. Although the transport of cultural relics is a mission for transport company, it still requires the museum party to cooperate. It also requires real-time monitoring of cultural relics in transit; determine the location and so on. On the transport way also can have the situation of the cultural relics damaged, anti-theft security needs to be considered.If a mistake happen in the process, may affect the museum's exhibition utilization rate, make the country lost a material culture treasure. However, in the traditional transportation process, the transfer of cultural relics and the inventory of cultural relics are generally managed, which can not be fully monitored and managed in the whole process. Therefore, the cultural relic transportation management system needs to solve the problems of cultural relic management in transit.

The cultural relic transportation management system still uses RFID technology. At the same time, because the transportation of cultural relics is long distance and large space scope, it is necessary to locate and manage cultural relics in transit based on GPS and GRPS technology. First of all, the handover of cultural relics to transport companies and the handover of cultural relics to the staff of the museum. In this transition, both sides of the staff through a ZigBee modules of the RFID electronic tag read/write device to handover cultural relics. And the related information transmission to the both sides of the track positioning management system. The management and monitoring of cultural relics are managed by their respective managers. In transit, a wireless positioning module based on GPS and GRPS technology is needed. The handover of cultural relics before transportation is finished, it is the transportation of cultural relics. In this process, GPS technology is used to locate the location of the cultural relics, and the positioning information is sent to the positioning tracking system through GPRS technology. In order to control the location information of cultural relics in real time. After the first transition of the transport company, the system will monitor the entire status of the transportation. When the cultural relic arrives at the destination for a second transition, the system will combine the information of RFID electronic tags transmitted and the positioning information sent back by GPRS. The mobile terminal of the manager shows the transportation status of the relics and the real-time monitoring information of the location.

\section{Summary}

The museum operates the IOT to transmit information between everything and everything, everything and human, endowing everything with human wisdom, and carrying out more efficient, scientific and comprehensive monitoring and management of cultural relics. The design and implementation of the four systems such as the temperature and humidity monitoring system, the location and tracking system of cultural relics, the storage management system of cultural relics and the transportation management system of cultural relics can provide great convenience for the museum's monitoring and management. The application of IOT technology has implemented the function of environmental monitoring and real-time dynamic monitoring of cultural relics, and effectively improves the work efficiency of monitoring and protection of cultural relics in museum. 


\section{Acknowledgement}

This research was financially supported by the 2015 Innovative Entrepreneurship Project of College Students in Hainan Province (No.20150126).

\section{References}

[1] T. Wang. Application of "Internet of Things" Technology to the Analysis and Management of Museum Collections - A case study of Emperor Qinshihuang's Mausoleum Site Museum [J]. Sciences of Conservation and Archaeology, 2014, Vol.26, No.1, p. 93-98.

[2] L.M. Wu, F.Y. Xu, H. Huang. The Demand Analysis of Museum Environment Monitoring and the Application of Internet of Things [J]. Sciences of Conservation and Archaeology, 2014, Vol.23, No.3, p. $96-102$.

[3] Z.C. Ji. Indoor Environmental Monitoring of Museum Based on IOT Technology [J]. Sciences of Conservation and Archaeology, 2014, Vol.23, No.3, p. 48-54. 